\title{
Effect of Root Flexibility on Bladed-Disk Dynamics
}

\author{
A.S. Mohamad and A.A.N. Al-Jawi \\ Department of Mechanical Engineering, King Abdulaziz University, P.O. Box 80204, Jeddah 21589, Saudi Arabia
}

(Received 18 July 2003; accepted 16 October 2003)

\begin{abstract}
Mode localisation in nearly periodic engineering structures has become one of the most important parameters in the dynamics of such structures. The bladed-disk assembly is one such structure with many practical applications. In this paper, the free mode localisation in a bladed-disk assembly is considered, where the blades are modelled with root flexibility using Green's functions. The roots consist of idealised rectangular blocks mounted on linear and rotational springs. The beams are then mono-coupled by linear springs to form the cyclic chain. It is found that two propagation bands appear in addition to those for beams with fixed roots. The frequencies at which these flexibility modes occur can be obtained by solving an eighth-order frequency polynomial whose coefficients depend only on the root flexibility parameters. Natural frequencies are calculated for tuned and mistuned cases using the Green's function formulation and the finite element method, and these are found to be in good agreement. The mode shapes corresponding to selected frequencies are computed using the finite element method. The effects of the root parameters on the mode localisation are also studied.
\end{abstract}

\section{INTRODUCTION}

In the dynamics of periodic (tuned) or nearly periodic (mistuned) structures, one of the most important parameters is the mode localisation factor, which measures the average exponential rate of vibration amplitude decay from one substructure to the next. The confinement of vibration energy in a small section of a mistuned system has been given due attention in the literature. The phenomenon of mode localisation was first predicted in solid-state physics by Anderson, ${ }^{1}$ while Hodges ${ }^{2}$ was the first to recognise that mode localisation can occur in engineering structures. Since the work of Hodges, numerous studies have been conducted in an attempt to understand the effects of mistuning on the dynamics of blade assemblies. The study of mode localisation in bladeddisk assemblies with fixed roots has received a great deal of attention. For instance Wei and Pierre ${ }^{3,4}$ examined both free and forced localised responses of mistuned cyclic assemblies. They introduced stochastic techniques to calculate the forced response statistics, while Pierre and $\mathrm{Cha}^{5}$ tackled localisation effects analytically in assemblies of multi-mode component systems and showed that confinement increases rapidly with frequency. It appears, however, that localisation in bladeddisk assemblies with flexible roots has not received as much attention, even though uncoupled blades with root flexibility have been investigated, for instance by Gront, ${ }^{6}$ Singh and Rawtani, ${ }^{7}$ Singh and Rawtani, ${ }^{8}$ and Singh. ${ }^{9}$ Bladed-disk assemblies, which in most models are intended to represent turbine blades on the periphery of a shaft, form an important part of the turbomachinery industry. Several methods are available for designing the blade root for mounting onto the shaft, including such slide-in types as the fir tree. These mounting methods provide for easy assembly and disassembly, accommodation of differential expansion between blade and rotor, balanced load distribution on blades and the provision of some degree of vibration damping. As the fixation of the blade to the disk provides for some amount of looseness, the effect of the fixation method on the frequencies and mode shapes of the system should be considered in the design of these units.

In this paper, we propose a mathematical model using Green's functions to study the dynamics of cyclic cantilever chains with root flexibilities. The root masses and springs, for expediency, are lumped into the Green's function. A vibration approach is then used to determine the localisation factors. A finite element code (ABAQUS) is also applied. The method of Green's functions was previously used by Nicholson and Bergman, ${ }^{10}$ Mohamad, ${ }^{11}$ and Mohamad and Al-Jawi, ${ }^{12}$ among others.

\section{MATHEMATICAL MODEL}

In the derivation of the mathematical model, reference is made to Fig. 1(a). $N$ beams are linearly coupled at $x=a$ by $N$ linear springs of constants $k_{1}, k_{2}, \ldots, k_{N}$. The beams and springs are numbered, as seen in the figure. The transverse displacement of the $n$-th beam, $y_{n}(x, t)$, is obtained by the Euler-Bernoulli beam theory, thus, for $N>n>1$,

$$
\begin{gathered}
E I_{n} \frac{\partial^{4} y_{n}(x, t)}{\partial x^{4}}+m_{n} \frac{\partial^{2} y_{n}(x, t)}{\partial t^{2}} \\
=\left[k_{n} y_{n+1}(x, t)-\left(k_{n}+k_{n-1}\right) y_{n}(x, t)+k_{n-1} y_{n-1}(x, t)\right] \delta(x-a),
\end{gathered}
$$

where $E I_{n}$ and $m_{n}$ are the flexural rigidity and mass per unit length, respectively. In Eq. (1), $\delta(x-a)$ is the Dirac delta function whose property relevant to this application is:

$$
\int_{-\infty}^{+\infty} f(x) \delta(x-a) d x=f(a) .
$$

As the vibrations are harmonic in time with frequency $\omega$, one may assume a solution to Eq. (1) of the form:

$$
y_{n}(x, t)=Y_{n}(x) \exp (i \omega t) .
$$

International Journal of Acoustics and Vibration, Vol. 8, No. 4, 2003 (pp 219-226) 